\title{
Evaluation de modèles de simulation des grandes échelles en canal plan turbulent chauffé asymétriquement : de la comparaison des grandeurs intégrales à l'analyse des corrélations
}

\section{Evaluation of large eddy simulation models in a turbulent channel flow asymmetrically heated : from integral values comparison to covariances analysis}

\author{
Martin DAVID ${ }^{1}$, Adrien TOUTANT ${ }^{1}$, Françoise BATAILLE ${ }^{1}$ \\ ${ }^{1}$ Laboratoire PROMES-CNRS (UPR 8521), Université de Perpignan via Domitia, Tecnosud-Rambla de la \\ thermodynamique, 66100 Perpignan - France, martin.david@ promes.cnrs.fr
}

\begin{abstract}
RÉSUMÉ. La présente étude concerne la simulation numérique des écoulements anisothermes dans les récepteurs solaires surfaciques à gaz sous-pression. Un écoulement d'air turbulent, dans un canal plan bi-périodique, à bas nombre de Mach et haut nombre de Reynolds de frottement est considéré. On effectue des tests a posteriori en vue de confronter les résultats obtenus par différents modèles de turbulence utilisés en simulation des grandes échelles à une simulation numérique directe. Des approches fonctionnelles, structurelles et mixte sont comparées. L'analyse porte sur des grandeurs intégrales liées à la dynamique et à la thermique de l'écoulement, des grandeurs moyennées, des écarts types de certaines d'entre elles. Des corrélations impliquant les vitesses et la température sont également étudiées.

ABSTRACT. This study deals with numerical simulation of anisothermal flows in gas pressurized solar receiver. A turbulent air flow, in a bi-periodical channel, at low Mach number and high Reynolds number is considered. A posteriori tests are performed in order to compare results of several turbulence models of large eddy simulations with a direct numerical simulation. Functional, structural and mixed models are evaluated. Dynamic and thermal integral values caracterizing the flow, as well as mean and fluctuating values of velocity and temperature are analysed.Velocity and temperature correlations are studied.

MOTS-CLÉS. Transferts de chaleur, Turbulence, Récepteurs solaires, Simulation numérique directe, Simulation des grandes échelles

KEYWORDS. Heat transfers, Turbulence, Solar Receiver, Direct numerical simulation, Large eddy simulation
\end{abstract}

\section{Nomenclature}

\begin{tabular}{|c|c|c|c|}
\hline Symbole & Signification, unités & Symbole & Signification, unités \\
\hline$C_{f}$ & coefficient de frottement & $\pi$ & tenseur sous-maille (corrélation masse volumique-vitesse) \\
\hline$C_{p}$ & capacité thermique, $\mathrm{J} \mathrm{kg}^{-1} \mathrm{~K}^{-1}$ & $\rho$ & masse volumique, $\mathrm{kg} \mathrm{m}^{-3}$ \\
\hline$f$ & frottement, $\mathrm{kg} \mathrm{m}^{-1} \mathrm{~s}^{-2}$ & $\tau$ & tenseur sous-maille (corrélation vitesse-vitesse) \\
\hline$L_{i}$ & taille du canal dans la direction $i, \mathrm{~m}$ & $\Phi$ & Flux de chaleur surfacique, $\mathrm{kW} \mathrm{m}^{-2}$ \\
\hline$\dot{m}$ & débit massique, $\mathrm{kg} \mathrm{s}^{-1}$ & \multicolumn{2}{|r|}{ Indices et exposants } \\
\hline$N_{i}$ & nombre de points dans la direction $i$ & $\tilde{\tau}$ & filtre de Favre \\
\hline$r$ & constante spécifique, $\mathrm{J} \mathrm{kg}^{-1} \mathrm{~K}^{-1}$ & $\overline{-}$ & filtre volumique \\
\hline$U_{c}$ & vitesse au centre du canal, $\mathrm{m} \mathrm{s}^{-1}$ & $b$ & bulk \\
\hline \multicolumn{2}{|r|}{ Symboles grecs } & $c h$ & côté chaud \\
\hline$\alpha$ & diffusivité thermique, $\mathrm{m}^{2} \mathrm{~s}^{-1}$ & $f r$ & côté froid \\
\hline$\delta$ & demi-hauteur du canal, $\mathrm{m}$ & $p$ & paroi \\
\hline$\Delta_{i}^{+}$ & taille de maille adimensionnée (direction $i$ ) & $r m s$ & écart type \\
\hline$\mu$ & viscosité dynamique, $\mathrm{Pa} \mathrm{s}$ & $\tau$ & frottement \\
\hline$\nu$ & viscosité cinématique, $\mathrm{m}^{2} \mathrm{~s}^{-1}$ & & \\
\hline
\end{tabular}


Le récepteur solaire des centrales à tour est un élément critique puisqu'il doit transférer la chaleur issue du rayonnement concentré vers le fluide caloporteur. Les récepteurs solaires actuels fonctionnent avec un cycle de Rankine. Le fluide utilisé avec cette génération de récepteurs est très majoritairement un sel fondu. La viscosité des sels fondus induit de fortes pertes de charge et nécessite de maintenir le fluide caloporteur au dessus de la température de fusion du sel pour entretenir un écoulement dans le circuit et en dessous de sa température de vaporisation. De plus, il est nécessaire d'ajouter un circuit supplémentaire visant à récupérer la chaleur du sel fondu pour ensuite alimenter une turbine et produire de l'électricité. Le fonctionnement avec deux circuits est source de pertes thermiques et de coûts supplémentaires. La nouvelle génération de récepteurs solaires fonctionne avec un cycle de Brayton. Le fluide caloporteur est un gaz pressurisé ou des particules ce qui permet de s'affranchir des limitations de température liées au fluide. Le fonctionnement à haut niveau de température augmente le rendement du cycle et donc la compétitivité de la technologie. Les travaux présentés dans cet article porte sur cette technologie. Le gaz retenu est de l'air pour des raisons de simplicité d'accès, de fonctionnement et de coût. L'optimisation de l'absorbeur solaire consiste à maximiser les échanges énergétiques tout en minimisant les pertes de charges dues au frottement avec les parois. Afin de permettre l'amélioration de ce composant il est fondamental de comprendre les phénomènes physiques complexes mis en jeu. Ces derniers sont liés au fort gradient de température entre les parois et au grand nombre de Reynolds caractérisant les écoulements dans le récepteur solaire. La simulation numérique de ces écoulements offre la possibilité d'appréhender leurs spécificités grâce à la résolution des équations de Navier-Stokes et d'énergie. Dans ces travaux, le récepteur solaire est assimilé à un canal plan bi-périodique. Cette géométrie simplifiée permet de reproduire les caractéristiques des écoulements au sein du système. Au prix d'un coût de calcul très important, les Simulations Numériques Directes (SND) fournissent des résultats de référence en résolvant la quasiintégralité du spectre de la turbulence. Les Simulations des Grandes Échelles (SGE), quant-à-elles, offrent un coût de calcul plus raisonnable grâce à l'introduction de modèles de turbulence dont le but est de reproduire les effets des petites échelles de turbulence, non calculées, sur les grandes. Il existe différents modèles de turbulence reposant sur des hypothèses variées. Pour juger la pertinence des modèles, il convient d'effectuer des comparaisons a posteriori des modèles de turbulence des SGE avec une SND [DTB 19b, DTB 19a]. Les tests a posteriori permettent d'évaluer les modèles en tenant compte de l'accumulation des erreurs numériques, de troncature et de modélisation [SAG 06].

De nombreuses études traitent des écoulements en canal plan bi-périodique mais la plupart sont effectuées en conditions isothermes [DTB 19a, MTN 04, TKM 04, BPB 08]. En outre, les travaux en situation anisotherme traitent majoritairement la température comme un scalaire passif [KAM 99]. Cela signifie que les effets de la température n'affectent pas l'écoulement. Récemment, des comparaisons entre des résultats de SGE et de SND en conditions anisothermes et en tenant compte des effets de dilatation ont été menées [DTB 19b, DTB 19c] mais elles sont opérées pour des simulations à nombre de Reynolds et niveaux de température faibles au regard des conditions rencontrées dans les récepteurs solaires des centrales à tour. À la connaissance des auteurs, les travaux présentés dans cet article comblent un manque dans la littérature en proposant une comparaison a posteriori des modèles de turbulence des SGE dans des conditions opératoires des centrales à tour en tenant compte du couplage entre la dynamique de l'écoulement et la température. L'objectif des travaux est d'apprécier la qualité des modèles dans ces conditions extrêmes. On jugera principalement la fidélité des résultats sur des grandeurs intégrales, moyennées et des écarts types de certaines de ces grandeurs liées à la dynamique et à la thermique 
de l'écoulement. Les corrélations de vitesse longitudinale et vitesse perpendiculaire aux parois ainsi que de vitesse longitudinale et température seront analysées.

Les équations de Navier-Stokes sous l'hypothèse d'un bas nombre de Mach et les modèles de turbulence sont présentés dans la section 2. La géométrie, le maillage, les conditions limites et la méthode numérique sont développés dans la section 3. Enfin, les résultats sont discutés dans la section 4.

\section{Résolution du problème}

\section{1. Équations de Navier-Stokes sous l'approximation bas nombre de Mach}

Les récepteurs solaires à gaz sous-pression sont caractérisés par un grand nombre de Reynolds et des niveaux de température élevés. Néanmoins la forte pression dans l'absorbeur solaire (10 bars) permet de conserver un nombre de Mach suffisamment faible pour appliquer l'approximation bas nombre de Mach proposée par Paolucci [PAO 82]. Le détail des équations utilisées est donné par Dupuy et al. [DTB 19a]. Cette approche consiste à considérer la compressibilité du fluide tout en négligeant les effets acoustiques. Elle repose sur le développement des variables des équations de Navier-Stokes en série du carré du nombre de Mach et aboutit à la séparation de la pression en pression thermodynamique, homogène dans l'espace et matérialisant la pression moyenne dans le récepteur; et en pression mécanique, dépendante de la vitesse du fluide et donc de la localisation dans le canal. Le gaz circulant dans le récepteur est de l'air. Il est assimilé à un fluide Newtonien respectant la loi des gaz parfaits. La capacité thermique à pression constante est prise à la moyenne des températures de parois et est considérée constante : $C_{p}=1155 \mathrm{~J}_{\mathrm{kg}} \mathrm{kg}^{-1} \cdot \mathrm{K}^{-1}$. Dans ces conditions, le nombre de Prandlt est de 0,87 . La constante spécifique de l'air, $r$, est déterminée grâce à la relation de Mayer et vaut $330 \mathrm{~J} \cdot \mathrm{kg}^{-1} \cdot \mathrm{K}^{-1}$. Les propriétés du fluide varient avec la température, en particulier, la viscosité dynamique qui suit la loi de Sutherland [SUT 93]. Lors des SGE, seule une partie du spectre de la turbulence est résolue. Un filtrage implicite est appliqué. Les échelles plus petites que la taille du filtre sont modélisées. Les deux termes sous-mailles étudiés par Dupuy et al. [DTB 19b] sont modélisés. Ils sont associés à la convection de la quantité de mouvement pour l'un et à la corrélation entre la masse volumique et la vitesse pour l'autre. Dans cette étude l'ensemble des modèles sont testés avec un filtre de Favre, noté $(\widetilde{\cdot})$, tel que $\widetilde{\phi}=\overline{\rho \phi} / \bar{\rho}$ pour toute variable $\phi$. L'intérêt de ce filtre réside dans la suppression des non-linéarités associées à la corrélation entre la masse volumique et la vitesse dans l'équation de conservation de la continuité. La formulation Favre engendre, néanmoins, des non-linéarités dans l'équation de conservation de l'énergie. Elle est souvent utilisée pour la résolution d'écoulements compressibles [GAS 09].

\subsection{Modèles de turbulence des Simulations des Grandes Échelles}

Les termes sous-mailles associés à la corrélation vitesse-vitesse, $\tau_{i j}=\widetilde{U_{j} U_{i}}-\widetilde{U_{j}} \widetilde{U_{i}}$, et à la corrélation entre la masse volumique et la vitesse, $\pi_{i j}=\widetilde{U_{j} / \rho}-\widetilde{U_{j}} / \widetilde{\rho}$, sont étudiés. Les modèles de turbulence des SGE sont construits à partir des propriétés connues de l'écoulement et visent à reproduire les effets des petites échelles de turbulence sur les grandes. On distingue les modèles fonctionnels des modèles structurels. Dans la suite les abréviations des modèles sont écrits entre parenthèses. Les valeurs des constantes utilisées sont celles recommandées par les auteurs des différentes approches. Elles suivent 
l'abréviation des modèles (le premier chiffre correspond aux unités puis les deuxième et troisième aux dixièmes et centièmes).

\section{Modèles fonctionnels}

Les modèles fonctionnels supposent un transfert d'énergie unilatéral des grandes structures vers les petites échelles par un processus analogue à la diffusion moléculaire. La remontée de la cascade d'énergie est alors négligée. La viscosité sous-maille, $\nu_{t}$, relie le tenseur sous-maille, $\tau_{i j}$, au tenseur des taux de déformation, $S_{i j}$. Son expression dépend du modèle utilisé.

$$
\tau_{i j}^{\text {mod }}=-2 \nu_{t}^{\text {mod }} S_{i j}
$$

On teste les modèles fonctionnels scalaires suivant : WALE (wal055) [NID 99], Sigma (sig100) [NTC 11] et AMD (amd030) [RBM 15]. Les expressions des viscosités sous-mailles sont listées ci-dessous :

$$
\nu_{t}^{W A L E}=\left(C^{W A L E} \bar{\Delta}\right)^{2} \frac{\left(S_{i j}^{d} S_{i j}^{d}\right)^{3 / 2}}{\left(S_{m n} S_{m n}\right)^{5 / 2}+\left(S_{m n}^{d} S_{m n}^{d}\right)^{5 / 4}}
$$

Où $S_{i j}^{d}$ est le déviateur du tenseur des taux de déformation.

$$
\nu_{t}^{\text {Sigma }}=\left(C^{\text {Sigma }} \bar{\Delta}\right)^{2} \frac{\sigma_{3}\left(\sigma_{1}-\sigma_{2}\right)\left(\sigma_{2}-\sigma 3\right)}{\sigma_{1}^{2}}
$$

Où $\sigma_{1}, \sigma_{2}$ et $\sigma_{3}$ sont les trois valeurs singulières du gradient de vitesse.

$$
\nu_{t}^{A M D}=C^{A M D} \frac{\max \left(0,-G_{i j} S_{i j}\right)}{g_{m n} g_{m n}}
$$

Où $G_{i j}={\overline{\Delta_{k}}}^{2} \partial_{k} U_{i} \partial_{k} U_{j}$ est le modèle de gradient pour le terme sous-maille associé à la convection de la quantité de mouvement. La version tensorielle, $H_{i j}^{(4)}$ proposée par Dupuy [DTB 19b], du modèle AMD pour la quantité de mouvement est également mise à l'épreuve afin de mieux tenir compte de l'anisotropie de l'écoulement. Elle est combinée au modèle AMD scalaire [ABM 16] pour la thermique. La combinaison AMD tensoriel-AMD scalaire est abrégée en AMD tensoriel (amdt030) dans ce qui suit pour des raisons de présentation.

\section{Modèles structurels}

Deux modèles structurels sont examinés : le modèle de gradient (grd100) [LEO 75] reposant sur un développement en série de Taylor du filtre et le modèle de similarité d'échelle (sim100) [BFR 80] supposant que la structure du tenseur construit sur la base des échelles sous-mailles est analogue à celle issue des plus petites échelles résolues. L'approche par similarité requiert l'application d'un filtre explicite. Dans cette étude, un filtre boîte, défini comme la moyenne de trois cellules dans les trois directions de l'espace, est utilisé. Le tenseur sous-maille associé à chacun de ces modèles est donné ci-dessous.

$$
\begin{aligned}
\tau_{i j}^{g r d} & =\frac{1}{12} C^{g r d}{\overline{\Delta_{k}}}^{2} \partial_{k} U_{i} \partial_{k} U_{j} \\
\tau_{i j}^{s i m} & =C^{s i m}\left({\widehat{U_{j} U_{i}}}-\widehat{U_{j}} \widehat{U}_{i}\right)
\end{aligned}
$$


On étudie un modèle couplant les deux stratégies présentées plus haut. Les modèles mixtes visent à tirer parti des avantages respectifs des deux méthodes. La modélisation fonctionnelle est généralement efficace pour déterminer les transferts d'énergie vers les petites échelles de turbulence. L'approche structurelle, quant-à-elle, donne une bonne estimation de la structure du tenseur sous-maille. Le modèle mixte multiplicatif (rds005) fondé sur le modèle de gradient, proposé par Dupuy [DTB 19b], est inclus dans les tests.

$$
\tau_{i j}^{r d s}=-C^{r d s} \frac{{\overline{\Delta_{k}}}^{2} \partial_{k} U_{k} \partial_{k} U_{k}}{|S|}
$$

\section{Configuration de l'étude}

La présente section décrit la géométrie et les conditions de simulation de l'étude dans un premier temps puis le maillage et les aspects numériques du problème dans un second temps.

\subsection{Géométrie et conditions de simulation}

Les directions longitudinale, $x$, et transversale, $z$, de l'écoulement sont périodiques. La taille du domaine est $L_{x}=4 \pi \delta, L_{y}=2 \delta$ et $L_{z}=4 / 3 \pi \delta$ avec $\delta=3 \mathrm{~mm}$ la demi-hauteur du canal. La face irradiée du récepteur solaire est associée à une paroi dont la température est fixée à $1300 \mathrm{~K}$. La température de la face opposée est imposée $900 \mathrm{~K}$ (voir figure 1). La circulation d'air entre les deux parois est maintenue en imposant un frottement au niveau du contact entre le solide et le fluide. Le nombre de Reynolds de frottement moyen vaut 800 . La pression thermodynamique dans le canal est de 10 bars.

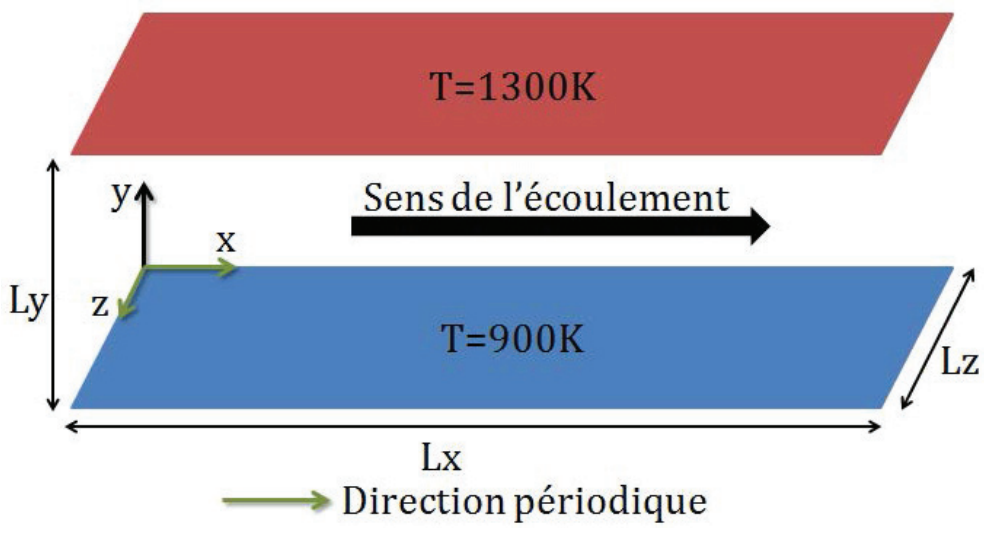

Figure 1. Canal plan

\subsection{Maillage et aspects numériques}

Les caractéristiques des maillages de la simulation numérique directe et des simulations des grandes échelles sont présentées dans le tableau 2.1. Elles contiennent respectivement 660 millions de cellules 


\begin{tabular}{ccc}
\hline & $N_{x} \times N_{y} \times N_{z}$ & $\Delta_{x}^{+} ; \Delta_{y}^{+}(0) ; \Delta_{y}^{+}(\delta) ; \Delta_{z}^{+}$ \\
\hline SND & $1152 \times 746 \times 768$ & 10,$4 ; 0,4 ; 5,2 ; 5,2$ \\
\hline SGE & $160 \times 152 \times 96$ & 74,$6 ; 1,0 ; 31,0 ; 41,5$ \\
\hline
\end{tabular}

Tableau 2.1. Caractéristiques des maillages de la simulation numérique directe et des simulations des grandes échelles

et 2,3 millions. Le coût de calcul de la SND est de 3,87 millions d'heures réparties sur 2304 cœurs. Les SGE nécessitent 23000 heures sur 48 cœurs.

Le maillage est régulier dans les deux directions homogènes et suit une loi en tangente hyperbolique dans la direction perpendiculaire aux parois :

$$
y_{k}=L_{y}\left(1+\frac{1}{a} \tanh \left[\left(\frac{k-1}{N_{y}-1}-1\right) \tanh ^{-1}(a)\right]\right)
$$

où $a$ est le paramètre de dilatation du maillage. Concernant les aspects numériques, on utilise une méthode aux différences finies pour approcher les différents termes des équations. Un schéma temporel Runge-Kutta d'ordre 3 est utilisé. Les opérateurs de convection et de diffusion sont centrés (d'ordre 4 pour la convection de la vitesse). La convection de la masse s'appuie sur un schéma Quick d'ordre 3. Nous avons recours à un solveur multigrille pour réduire le temps de résolution du calcul de la pression : la solution de l'équation de Poisson est calculée sur des maillages de plus en plus précis, en utilisant comme condition initiale le résultat du niveau de grille précédent. Le code de calcul utilisé est TrioCFD [CCE 02].

\section{Résultats et discussion}

Dans ce qui suit les résultats de la simulation numérique directe et des simulations des grandes échelles sont comparés. Le domaine d'étude est bi-périodique dans les directions longitudinale et transversale de l'écoulement. Les résultats obtenus sont intégrés suivant les directions périodiques. Les graphiques tracés sont donc indépendants de la position $x$ dans le canal. On distingue les grandeurs intégrales, les grandeurs moyennées temporellement et les écarts types des grandeurs. Sauf indication contraire, le modèle de SGE annoncé est utilisé pour les deux termes sous-mailles modélisés (la corrélation vitesse-vitesse, $\tau_{i j}$, et la corrélation masse volumique vitesse, $\pi_{i j}$ ). Les résultats d'une $\mathrm{SGE}$ sans modèle sont également présentés pour mettre en exergue les effets des modèles.

\subsection{Grandeurs intégrales}

Cette partie présente des résultats globaux obtenus par les différents modèles de simulation des grandes échelles. Le débit, les nombres de Reynolds débitant, $R e_{b}=2 \delta U_{b} / \nu_{b}$, et de frottement, $R e_{\tau}=\delta U_{\tau} / \nu_{p}$, le flux et la vitesse débitante sont exposés dans le tableau 3.2.

Tout d'abord, les résultats montrent que le débit obtenu par les modèles des SGE est proche de celui de la SND. Le frottement étant imposé, les imprécisions liées à la modélisation des phénomènes en proche paroi se répercutent sur le débit. Ces écarts sont faibles puisqu'ils sont inférieurs à $7 \%$ dans tous les cas. Ensuite, l'analyse des valeurs de flux pariétaux révèle que toutes les SGE sous-estiment les transferts de 
chaleur entre le solide et le fluide. Les meilleurs résultats sont obtenus avec la combinaison des modèles AMD tensoriel pour la dynamique et AMD scalaire pour la thermique (amdt030). L'écart est alors de $10 \%$ environ. L'approche par similarité d'échelle donne de très bons résultats concernant l'estimation de la vitesse débitante.

\begin{tabular}{rcccccc}
\hline & $\dot{m}$ & $R e_{b}$ & $R e_{\tau, f r}$ & $R e_{\tau, c h}$ & $\Phi$ & $U_{b}$ \\
\hline SND & 0,0164 & 29979 & 968 & 625 & 98,4 & 79,7 \\
\hline sans modèle & 0,0161 & 29118 & 980 & 625 & 80,0 & 77,2 \\
\hline AMD (amd030) & 0,0177 & 32215 & 970 & 630 & 88,8 & 85,4 \\
\hline AMD tensoriel (amdt030) & 0,0158 & 28659 & 973 & 627 & 89,3 & 76,0 \\
\hline Sigma (sig100) & 0,0171 & 31096 & 974 & 628 & 86,5 & 82,4 \\
\hline WALE (wal055) & 0,0169 & 30732 & 975 & 628 & 86,2 & 81,5 \\
\hline Mixte multiplicatif (rds005) & 0,0162 & 29393 & 979 & 624 & 81,9 & 77,9 \\
\hline Similarité d'échelle (sim100) & 0,0166 & 30034 & 970 & 628 & 81,1 & 79,6 \\
\hline Gradient (grd100) & 0,0157 & 28565 & 979 & 624 & 80,1 & 75,7 \\
\hline & & & & & &
\end{tabular}

Tableau 3.2. Ecarts entre les résultats SND et SGE sur des grandeurs intégrales, partie 1

\begin{tabular}{rccccc}
\hline & $U_{c} / U_{b}$ & $U_{b} / U_{\tau, f r}$ & $U_{b} / U_{\tau, c h}$ & $C_{f, f r}$ & $C_{f, c h}$ \\
\hline SND & 1,135 & 21,04 & 18,14 & $4,52 \times 10^{-3}$ & $6,08 \times 10^{-3}$ \\
\hline sans modèle & 1,138 & 20,43 & 17,83 & $4,79 \times 10^{-3}$ & $6,29 \times 10^{-3}$ \\
\hline AMD (amd030) & 1,116 & 22,85 & 19,56 & $3,83 \times 10^{-3}$ & $5,23 \times 10^{-3}$ \\
\hline AMD tensoriel (amdt030) & 1,127 & 20,21 & 17,46 & $4,90 \times 10^{-3}$ & $6,56 \times 10^{-3}$ \\
\hline Sigma (sig100) & 1,119 & 21,95 & 18,95 & $4,15 \times 10^{-3}$ & $5,56 \times 10^{-3}$ \\
\hline WALE (wal055) & 1,120 & 21,70 & 18,73 & $4,25 \times 10^{-3}$ & $5,70 \times 10^{-3}$ \\
\hline Mixte multiplicatif (rds005) & 1,134 & 20,62 & 17,99 & $4,71 \times 10^{-3}$ & $6,18 \times 10^{-3}$ \\
\hline Similarité d'échelle (sim100) & 1,138 & 21,26 & 18,27 & $4,43 \times 10^{-3}$ & $5,99 \times 10^{-3}$ \\
\hline Gradient (grd100) & 1,146 & 20,03 & 17,49 & $5,00 \times 10^{-3}$ & $6,54 \times 10^{-3}$ \\
\hline & & & & &
\end{tabular}

Tableau 3.3. Ecarts entre les résultats SND et SGE sur des grandeurs intégrales, partie 2

Dans le tableau 3.3, le coefficient de frottement est défini à partir de la vitesse débitante et des grandeurs pariétales :

$$
C_{f, p}=2 f_{p} / \rho_{p} U_{b}^{2} .
$$

Le modèle mixte multiplicatif proposé par Dupuy [DTB 19b] est celui qui prédit le mieux le rapport des vitesses $U_{c} / U_{b}$ (voir tableau 3.3). Le modèle de similarité d'échelle détermine les coefficients de friction avec précision. Les corrélations empiriques de Dean [DEA 78] permettent d'estimer le rapport $U_{c} / U_{b}$ :

$$
\frac{U_{c}}{U_{b}}=1,28 R e_{b}^{-0,0116}
$$

et le coefficient de friction :

$$
C_{f}=0,073 R e_{b}^{-1 / 4}
$$


On obtient alors $U_{c} / U_{b}=1,136$ et $C_{f}=5,55 \times 10^{-3}$. Le rapport des vitesses issu de la corrélation de Dean est très proche des valeurs de la SND et est meilleur que tous les résultats obtenus par les modèles des SGE (le modèle mixte multiplicatif mis à part). En définissant un nombre de Reynolds dépendant du côté, $R e_{b, p}=2 \delta U_{b} \rho_{p} / \mu_{p}$, il est possible de déterminer un coefficient de friction pour chaque paroi à l'aide de cette corrélation. On obtient alors $C_{f \text { Dean, } f r}=5,13 \times 10^{-3}$ et $C_{f \text { Dean,ch }}=5,94 \times 10^{-3}$. On note que les valeurs obtenues par la corrélation dépendante du côté sont plus grande côté froid et plus petite côté chaud que celles issues de la SND $\left(C_{f S N D, f r}<C_{f D e a n, f r}<C_{f D e a n, c h}<C_{f S N D, c h}\right)$.

\subsection{Grandeurs moyennes}

Les profils des valeurs, moyennées temporellement et spatialement dans les directions longitudinale et transversale, de vitesse longitudinale adimensionnée et température des différentes simulations sont exposés sur les figures 2 et 3 . Les résultats sont comparés à la SND. Rappelons toutefois que, en toute rigueur, les résultats des simulations des grandes échelles ne permettent pas de retrouver les valeurs des SND mais les valeurs des SND filtrées.

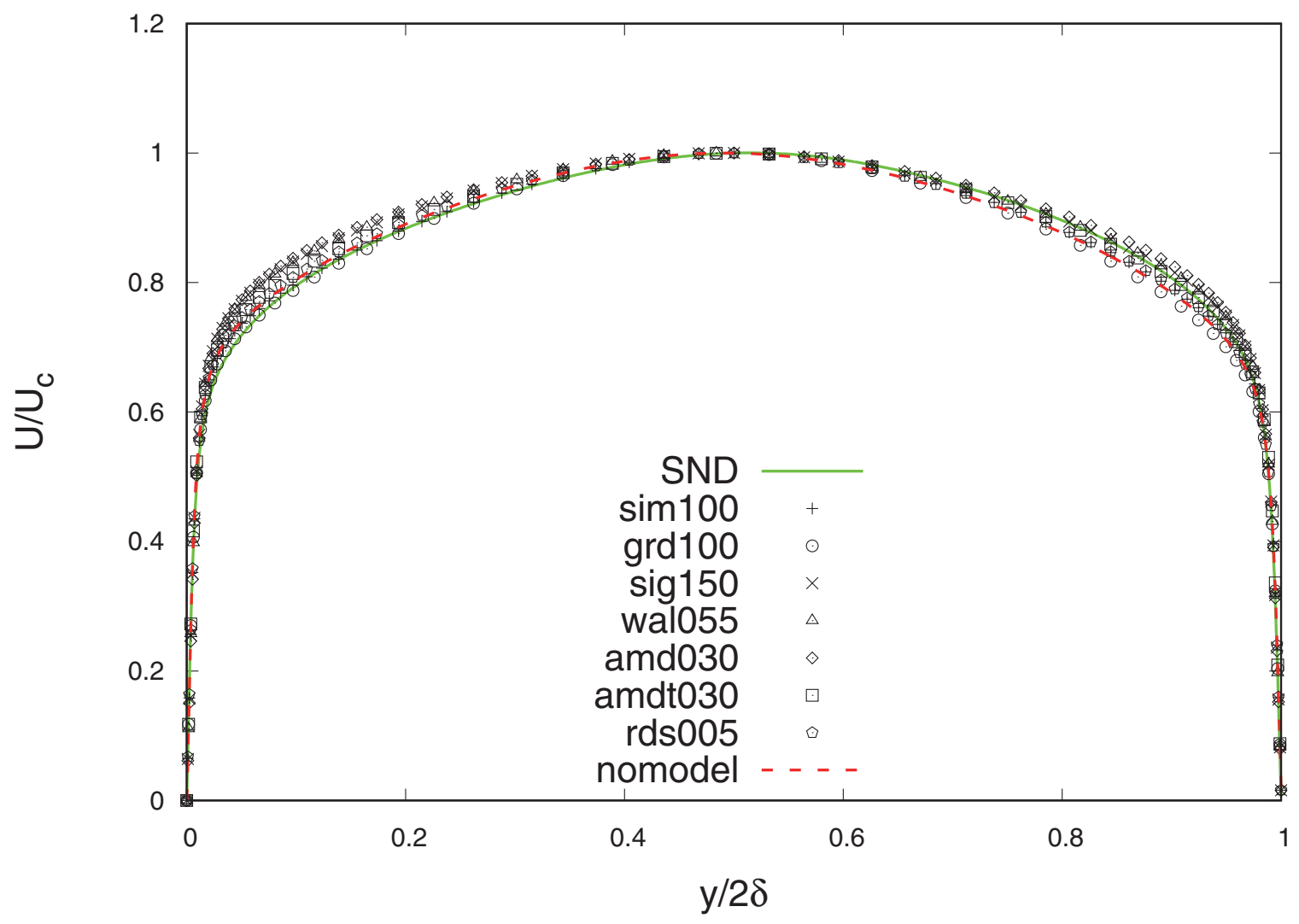

Figure 2. Profil de vitesse adimensionnée

Les courbes de vitesse adimensionnées sont caractéristiques d'un écoulement turbulent car elles présentent de forts gradients de vitesse à proximité des parois. Bien que présente, l'asymétrie du profil de vitesse de SND reste faible en raison du fort nombre de Reynolds. Les résultats obtenus par SGE et SND sont très similaires. Néanmoins l'asymétrie du profil de vitesse, liée aux gradients de température, de la SND n'est pas reproduite par les SGE. Les profils de température (figure 3) traduisent un écoulement 


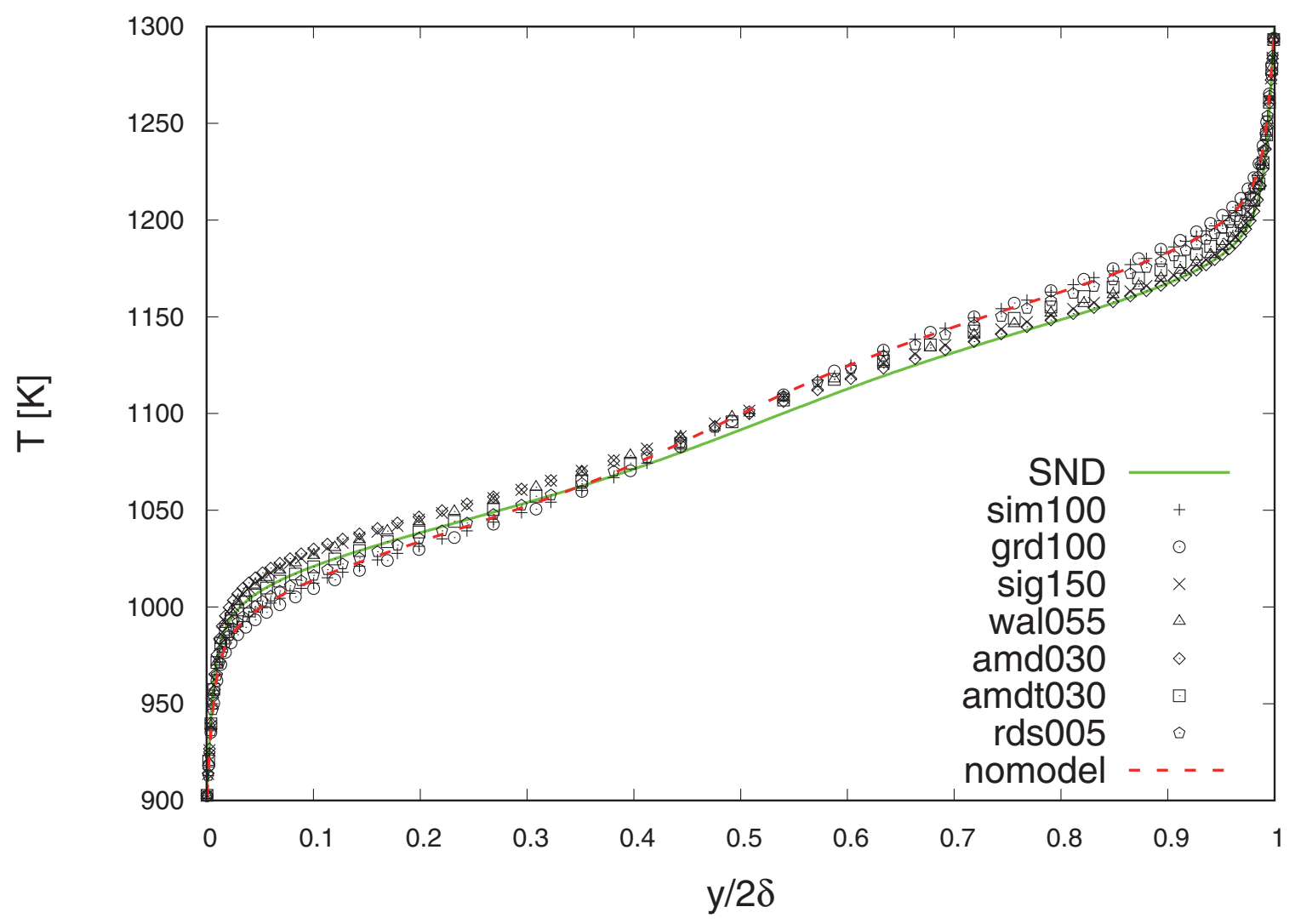

Figure 3. Profil de température

soumis à un fort flux. En effet, les gradients de température en proche paroi sont très forts. La température moyenne du fluide est proche de la moyenne des températures de paroi conformément aux valeurs obtenues pour un récepteur solaire infiniment long. La température au centre du canal est légèrement inférieure à $1100 \mathrm{~K}$ ce qui s'explique par l'influence de la dilatation thermique sur la dynamique de l'écoulement. Les profils de température des SGE suivent le comportement des résultats de la SND. Les SGE ont tendance à légèrement surestimer la température moyenne. Tous les écarts obtenus restent inférieurs à 4\%. Côté chaud, l'introduction d'un modèle de turbulence est bénéfique. Le modèle AMD donne des résultats satisfaisants à haute température. Au niveau de la paroi froide, le modèle AMD tensoriel, combiné à l'approche AMD scalaire pour la thermique, est le plus convaincant.

\section{3. Écarts types des grandeurs}

On compare, ici, les profils d'écarts types de vitesse et de température des simulations des grandes échelles avec ceux de la simulation numérique directe (voir figures 4 et 5).

Les profils d'écarts types de vitesse longitudinale (voir figure 4) montrent l'apparition d'un pic en proche paroi de chaque face du récepteur. Ils sont localisés dans la zone tampon de la couche limite et sont associés à des structures très allongées dans la direction longitudinale appelées streaks. Le pic côté chaud est supérieur de 9\% au pic côté froid. Les profils d'écarts types de vitesse montrent que toutes les valeurs issues des SGE sont supérieures aux résultats de la SND au niveau des parois. Les modèles AMD tensoriel et similarité d'échelle sont les plus satisfaisants à proximité de ces parois. Concernant le 


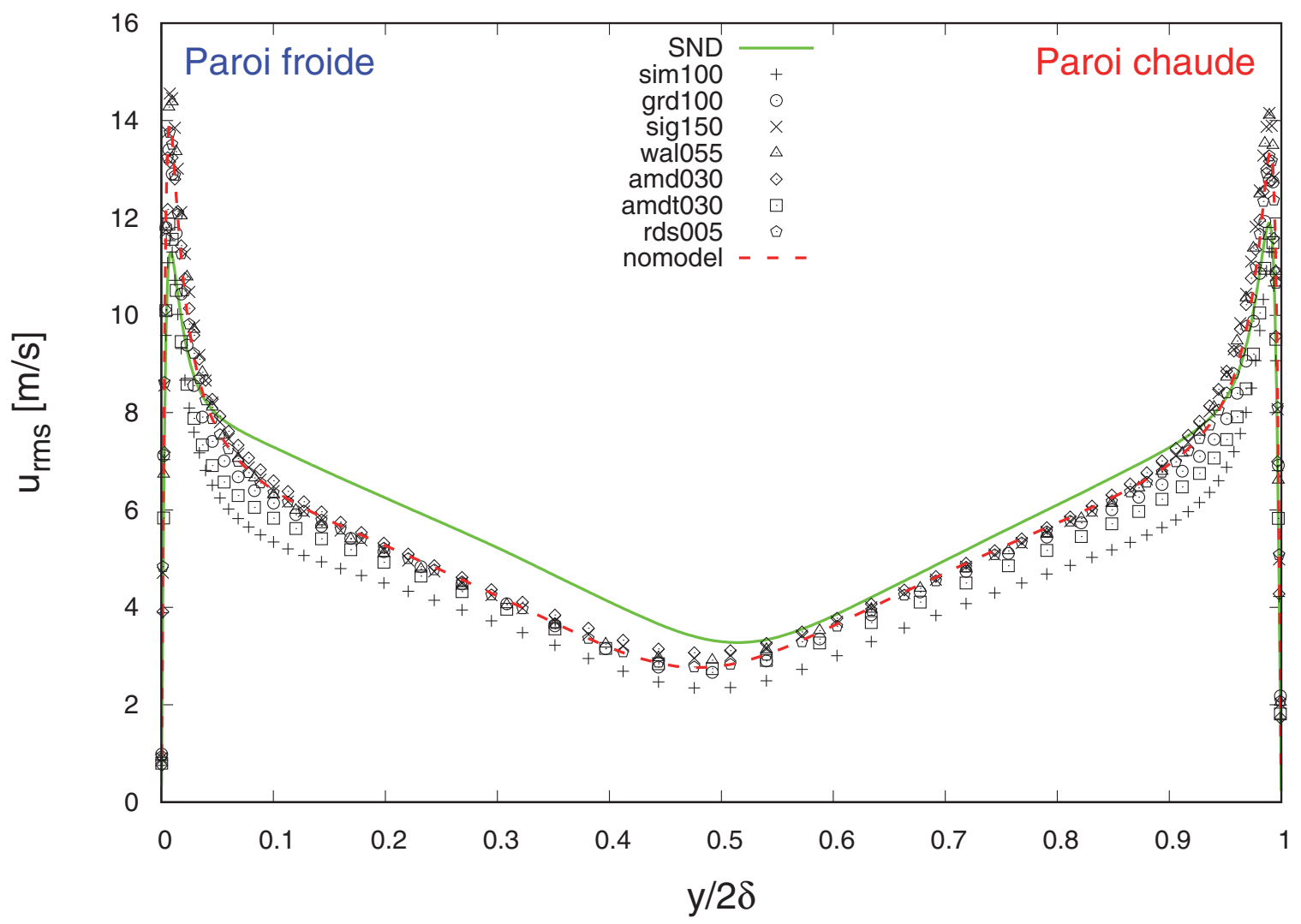

Figure 4. Profil d'écart type de vitesse

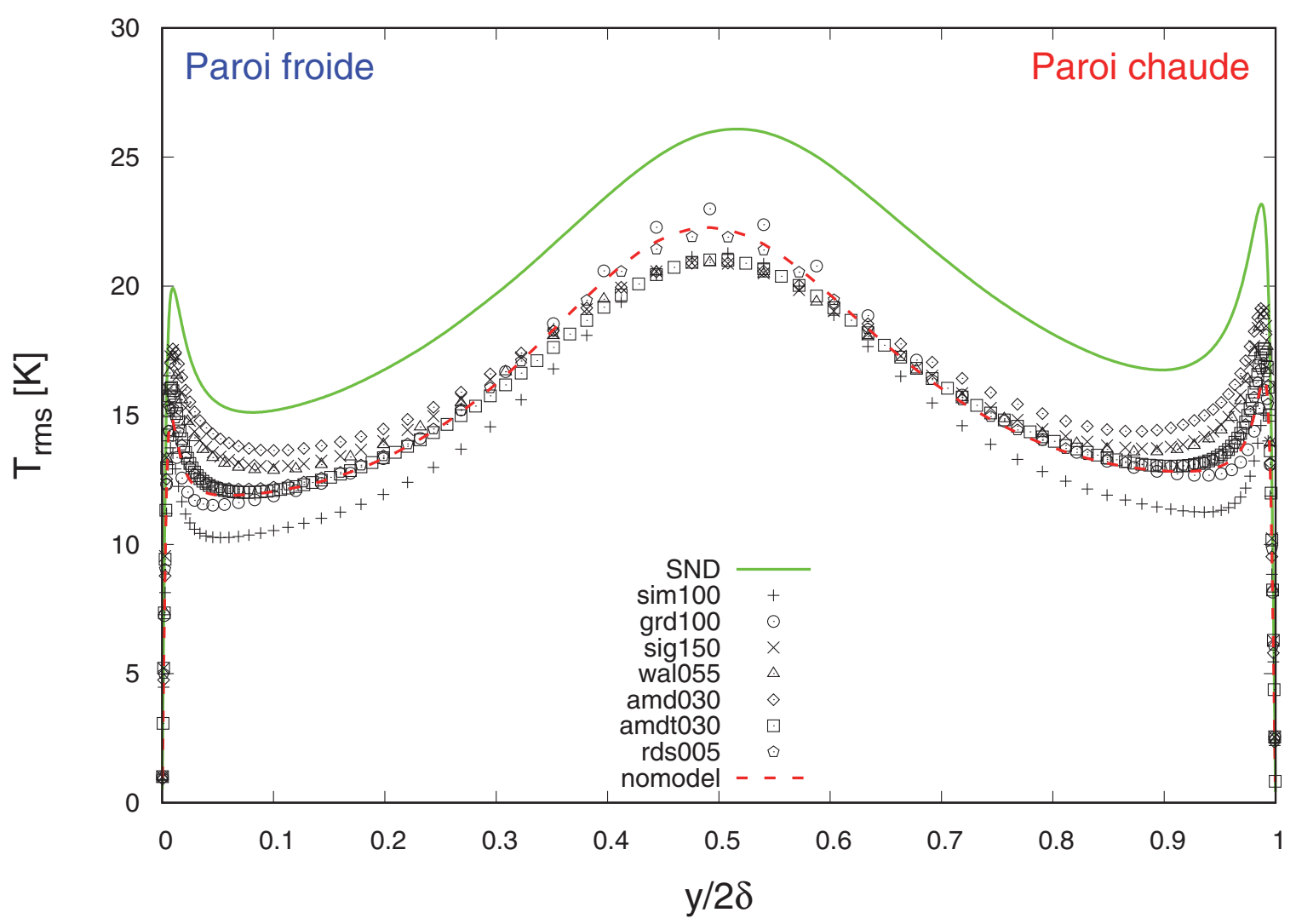

Figure 5. Profil d'écart type de température 
profil d'écart type de température (figure 5), les résultats de la SND décrivent un profil constitué d'un pic en proche paroi pour chaque côté. Comme pour les fluctuations de vitesse, ces pics sont localisés dans la zone tampon. Le pic côté chaud est plus important que celui situé en proche paroi froide. En outre, un pic central, supérieur aux pics pariétaux, est observé. Il est légèrement décalé vers le côté froid $(y / 2 \delta=0,52)$. Ce pic pourrait être associé aux grandes structures turbulentes traversant la région centrale du récepteur solaire. Les valeurs de la SND sont sous-estimées par les SGE. Les estimations sont globalement meilleures au niveau de la paroi froide. Bien que loin des résultats de la SND, les modèles sigma, AMD et WALE sont les plus performants. À l'inverse, les modèles AMD tensoriel, similarité d'échelle et gradient présentent des résultats insatisfaisants. Le reste des modèles est plus performant que la simulation sans modèle.

\subsection{Corrélations}

La pertinence des modèles est étudiée sur des corrélations faisant intervenir le produit de la fluctuation de vitesse longitudinale et de la fluctuation de vitesse perpendiculaire aux parois (figure 6) ainsi que le produit de la fluctuation de vitesse longitudinale et de la fluctuation de température (figure 7).

Pour analyser les résultats, l'adimensionnement classique $\left(^{+}\right)$est utilisé :

$$
\left(u^{\prime} v^{\prime}\right)^{+}=<u^{\prime} v^{\prime}>\frac{1}{U_{\tau}^{2}}
$$

et

$$
\left(u^{\prime} T^{\prime}\right)^{+}=<u^{\prime} T^{\prime}>\frac{\rho C_{p}}{\Phi_{p}} .
$$

La figure 6 décrit l'évolution de la corrélation $\left\langle u^{\prime} v^{\prime}>\right.$ suivant la direction perpendiculaire aux parois. Les pics sont localisés au début de la zone logarithmique de la couche limite. Dans cette zone, les "jambes" des vortex appelés hairpins commencent à se redresser pour former des rouleaux dont l'axe de rotation est perpendiculaire aux parois. Ces phénomènes tourbillonnaires peuvent être à l'origine des pics de la corrélation $\left\langle u^{\prime} v^{\prime}\right\rangle$. Là encore, on compare les résultats des SGE aux données de SND. Le profil est bien reproduit par les modèles, excepté l'approche par similarité d'échelle qui sous estime les pics de la corrélation SND d'environ 40\%. A proximité de la paroi chaude, les calculs WALE, mixte multiplicatif et sans modèle sont très satisfaisants. Côté froid, les simulations avec les modèles WALE, mixte multiplicatif, sigma et sans modèle sont les plus performantes.

La figure 7 décrit l'évolution du profil de la corrélation $\left\langle u^{\prime} T^{\prime}\right\rangle$. Les résultats de SND révèlent la présence de deux pics situés en proche paroi, dans la zone tampon. Le profil de la simulation numérique directe est bien reproduit par la majorité des modèles. Côté froid, les modèles fonctionnels mixte multiplicatif et WALE sont très satisfaisants ainsi que l'approche structurelle du gradient. En proche paroi chaude, WALE et AMD sont les meilleurs. Les modèles sigma et similarité d'échelle se démarquent, respectivement, en surestimant et sous-estimant les valeurs extrêmes.

\section{Conclusion}

Une simulation numérique directe et plusieurs simulations des grandes échelles ont été effectuées à grand nombre de Reynolds, niveau de température élevé et fort flux. Les équations de Navier-Stokes sont 


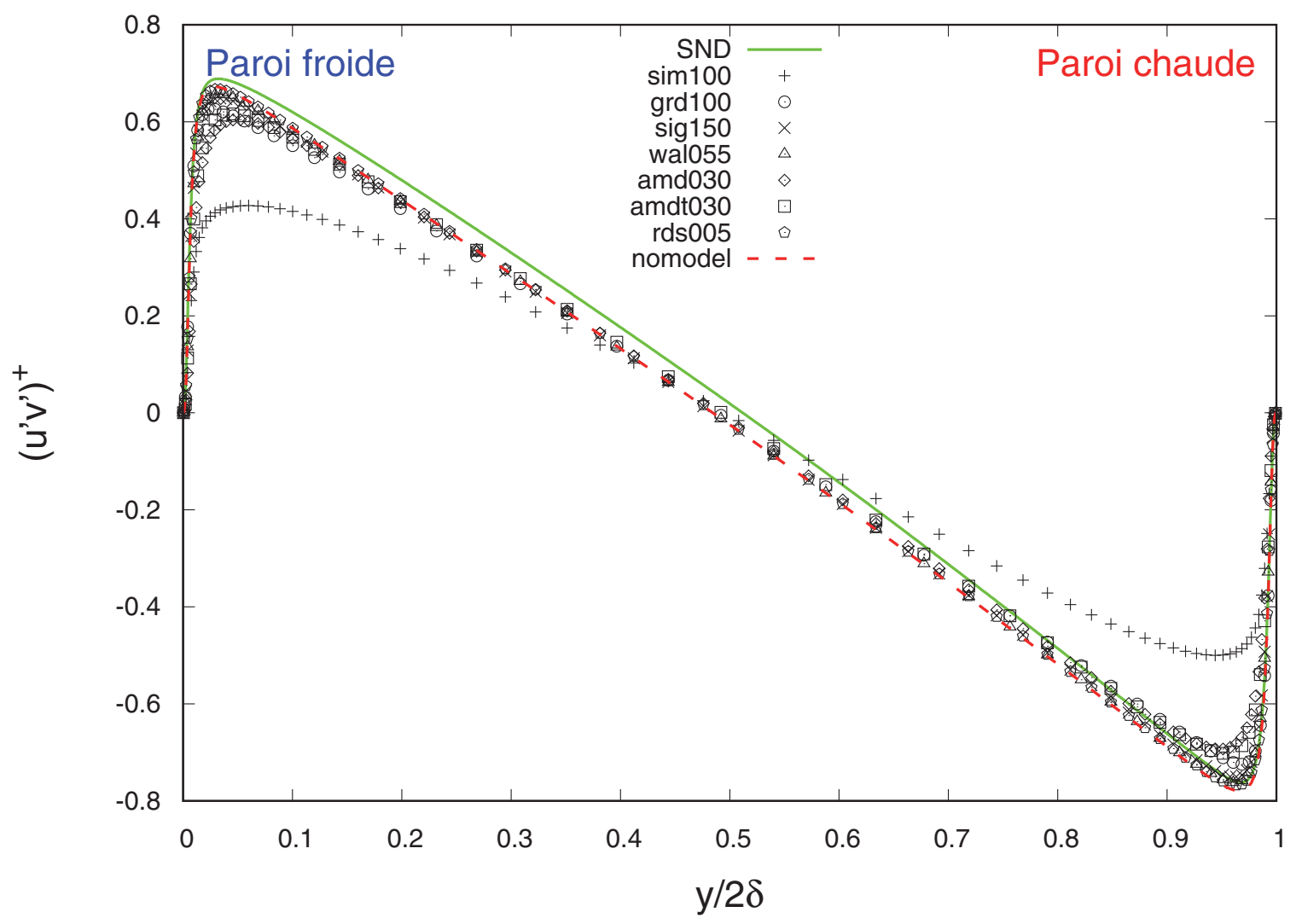

Figure 6. Corrélation de vitesse longitudinale et vitesse perpendiculaire aux parois

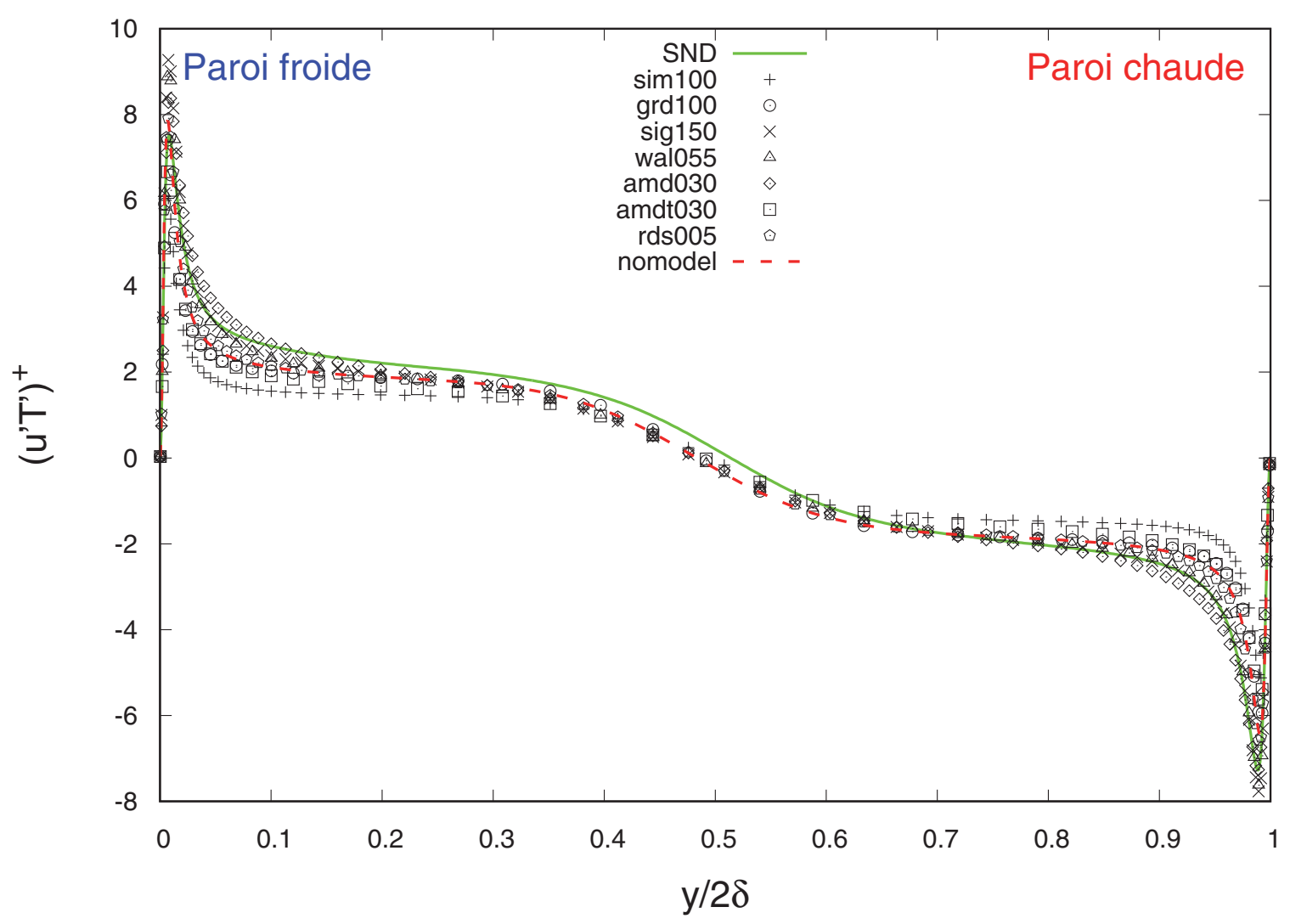

Figure 7. Corrélation de vitesse longitudinale et température 
résolues sous l'hypothèse bas nombre de Mach en vue de reproduire les conditions de fonctionnement des récepteurs solaires des centrales à tour. Le couplage entre la dynamique et les effets de la température est pris en compte. Les résultats obtenus montrent que les modèles de turbulence testés parviennent à retranscrire les profils de vitesse et de température ainsi que le débit de façon très satisfaisante. On note que toutes les SGE sous-estiment les flux de chaleur pariétaux. Les profils d'écarts types de vitesse et de température sont plus difficilement estimés. Hormis pour les corrélations, il semble que le modèle similarité d'échelle soit le plus performant pour déterminer la dynamique de l'écoulement. La thermique est mieux prédite par le modèle AMD scalaire. Enfin, le modèle WALE prévaut pour l'estimation des corrélations.

\section{Bibliographie}

[ABM 16] Mahdi Abkar, Hyun J. Bae, and Parviz Moin. Minimum-dissipation scalar transport model for large-eddy simulation of turbulent flows. Phys. Rev. Fluids, 1(4):041701, 2016.

[BFR 80] J. Bardina, J. Ferziger, and W. Reynolds. Improved subgrid-scale models for large-eddy simulation. In 13th Fluid and PlasmaDynamics Conference. American Institute of Aeronautics and Astronautics, 1980.

[BPB 08] Christophe Brun, Margareta Petrovan Boiarciuc, Marie Haberkorn, and Pierre Comte. Large eddy simulation of compressible channel flow. Theor. Comput. Fluid Dyn., 22(3) :189-212, 2008.

[CCE 02] Christophe Calvin, Olga Cueto, and Philippe Emonot. An object-oriented approach to the design of fluid mechanics software. ESAIM : Mathematical Modelling and Numerical Analysis - Modélisation Mathématique et Analyse Numérique, 36(5) :907-921, 2002.

[DEA 78] Dean, R. B. Reynolds number dependence of skin friction and other bulk flow variables in two-dimensional rectangular duct flow. J. Fluids Eng, 100(2) :215, 1978.

[DTB 17] Dorian Dupuy, Adrien Toutant, and Françoise Bataille. Étude de l'équation d'énergie pour le développement de modèles sous-mailles adaptés aux écoulements fortement anisothermes. In $25^{\text {eme }}$ Congrès Français de Thermique, page 8. SFT, 2017.

[DTB 19a] D. Dupuy, A. Toutant, and F. Bataille. A posteriori tests of subgrid-scale models in an isothermal turbulent channel flow. Physics of Fluids, 31(4):045105, 2019.

[DTB 19b] D. Dupuy, A. Toutant, and F. Bataille. A posteriori tests of subgrid-scale models in strongly anisothermal turbulent flows. Physics of Fluids, 31(6):065113, 2019.

[DTB 19c] Dorian Dupuy, Adrien Toutant, and Françoise Bataille. A priori tests of subgrid-scale models in an anisothermal turbulent channel flow at low mach number. International Journal of Thermal Sciences, 145 :105999, 2019.

[GAS 09] E Garnier, N Adams, and P Sagaut. Large Eddy Simulation for Compressible Flows. Springer Science \& Business Media edition.

[KAM 99] Hiroshi Kawamura, Hiroyuki Abe, and Yuichi Matsuo. DNS of turbulent heat transfer in channel flow with respect to Reynolds and Prandtl number effects. International Journal of Heat and Fluid Flow, 20(3) :196-207, 1999.

[LEO 75] A. Leonard. Energy cascade in large-eddy simulations of turbulent fluid flows. 18 :237-248, 1975.

[MTN 04] Y. Morinishi, S. Tamano, and K. Nakabayashi. Direct numerical simulation of compressible turbulent channel flow between adiabatic and isothermal walls. Journal of Fluid Mechanics, 502 :273-308, 2004.

[NID 99] F. Nicoud and F. Ducros. Subgrid-scale stress modelling based on the square of the velocity gradient tensor. Flow, Turbulence and Combustion, 62(3) :183-200, 1999.

[NTC 11] Franck Nicoud, Hubert Baya Toda, Olivier Cabrit, Sanjeeb Bose, and Jungil Lee. Using singular values to build a subgrid-scale model for large eddy simulations. Physics of Fluids, 23(8) :085106, 2011.

[PAO 82] Samuel Paolucci. On the filtering of sound from the navier-stokes equations. NASA STI/Recon Technical Report $N, 83$ :26036, 111982.

[RBM 15] Wybe Rozema, Hyun J. Bae, Parviz Moin, and Roel Verstappen. Minimum-dissipation models for large-eddy simulation. Physics of Fluids, 27(8) :085107, 2015.

[SAG 06] Sagaut Pierre. Large Eddy Simulation for Incompressible Flows. Springer Science \& Business Media edition, 2006. 
[SUT 93] William Sutherland. The viscosity of gases and molecular force. The London, Edinburgh, and Dublin Philosophical Magazine and Journal of Science, 36(223) :507-531, 1893.

[TKM 04] M. Tanahashi, S. J. Kang, T. Miyamoto, S. Shiokawa, and T. Miyauchi. Scaling law of fine scale eddies in turbulent channel flows up to $R e_{\tau}=800$. International Journal of Heat and Fluid Flow, 25(3) :331-340, 2004.

\section{Remerciements}

Ces travaux ont bénéficié d'un accès aux moyens de calcul du CINES au travers de l'allocation de ressources A0062A05099 attribuée par GENCI (Grand Équipement National de Calcul Intensif). Nous remercions le CEA pour la mise à disposition de la plateforme TRUST et le développement du code de calcul TrioCFD (open source). Nous remercions également la région Occitanie pour leur participation au financement de ces travaux de thèse. 